\title{
Saturation des urgences : parallèle et paradoxe
}

\section{Emergency Department overcrowding: parallel and paradox}

\author{
Y. Freund \\ (C) SFMU et Springer-Verlag France 2013
}

Parlant de la circulation routière, un encombrement se forme lorsque l'affluence est supérieure à la capacité de la route. Cela dit, il convient de distinguer deux causes qui paraissent bien distinctes : d'une part, l'augmentation du trafic (aux heures de pointes par exemple) amène une saturation des capacités créant ainsi le ralentissement ; d'autre part, un évènement ponctuel ralentissant la circulation crée une diminution des capacités, et aura des conséquences similaires.

$\mathrm{Si}$ on reprend ces deux causes qu'on a pour habitude de distinguer, on peut remarquer que le mécanisme est le même :

- dans les deux cas, l'affluence dépasse les capacités ;

- dans les deux cas, le ralentissement crée l'encombrement.

L'analogie avec les services d'urgences est immédiate. La saturation de ceux-ci est un enjeu majeur de notre spécialité. Dans un article évaluant les perspectives de la médecine d'urgence, Sklar et al. expliquent que « Les access block et la saturation des urgences ont créé une tension dynamique, dont la résolution déterminera l'avenir de la médecine d'urgence »[1]. Comme pour le trafic routier, on identifie classiquement deux causes à la saturation des services d'urgences : une affluence importante, et un ralentissement dans la prise en charge ou l'aval des urgences. Ce dernier est bien connu sous l'anglicisme "Access block» [2], et est reconnu comme le grand pourvoyeur de saturation des services d'accueil des urgences (SAU). De même, la saturation des services amène le blocage. La diminution des lits d'aval des urgences, face à une demande sans cesse croissante, demeure le principal problème et la cause centrale de ces acces block. Elle rend au passage illusoire toute volonté de régler le problème de la saturation des urgences en ne proposant que des solutions centrées sur les SAU. Faciliter l'accès à l'hospitalisation d'aval doit rester une priorité.

\footnotetext{
Y. Freund $(\bowtie)$

Service d'accueil des urgences, GH Pitié-Salpêtrière,

Assistance publique-hôpitaux de Paris,

et université Pierre et Marie Curie,

47-83, boulevard de l'Hôpital,

F-75651 Paris cedex 13, France

e-mail : yonatman@gmail.com
}

L'augmentation du recours au SAU, les access block (attente pour un scanner, attente pour biologie ou un lit par exemple), l'arrivée de malades graves dont il faut s'occuper sans délai, l'affluence aux heures de pointe, etc. sont problématiques.

De multiples interventions ont été intentées pour enrayer ces difficultés majeures et ont ainsi contribué à diminuer le temps d'attente et de passage aux urgences. On citera la mise en place de structures d'observation, l'augmentation du nombre de personnel paramédical, l'augmentation du nombre de médecins, le redéploiement des effectifs à des horaires décalés et adaptés [3-8].

Pour soulager le traffic routier aux heures de pointes, peuvent être ouvertes des voies auxiliaires sur les autoroutes, ou des itinéraires bis. De même, certains SAU ont mis en place avec succès des « circuits courts » de consultations simples aux heures les plus chargées, afin de diminuer la charge de travail globale et délester le flux entrant. Tout ceci mérite une analyse bien plus poussée que ce que l'on fait ici, et il est du devoir de nos institutions, centres hospitaliers, sociétés savantes, de poursuivre les efforts et les recherches dans le but de rendre plus fluide l'accès au soin via un SAU. Après tout, le temps d'attente est inversement corrélé à un bon pronostic [9].

Le paradoxe est le suivant : sur le périphérique aux heures de pointes, le débit est moindre alors qu'il y a plus de voitures. Si on y regarde de plus près, il n'y a rien de trivial là dedans. Habituellement, quand un fluide s'écoule, plus la densité est élevée, plus le débit est élevé. De manière plus intéressante encore, la conservation de l'énergie pour un écoulement prévoit que la réduction de la section de passage (un entonnoir en somme, belle image d'un SAU) a pour conséquence directe une augmentation de la vitesse. Tout du moins jusqu'à une certaine limite, après quoi l'écoulement devient turbulent. Cette limite est une réalité bien appréhendée dans les applications que nous évoquons. Le périphérique, comme un SAU, a une capacité limite. Une fois les capacités débordées, c'est-à-dire au delà d'un certain débit d'entrée, on sort de ce qu'on appelle le « régime stationnaire », i.e. le débit de sortie est égal au débit d'entrée. 
Ainsi, il existe un seuil au-delà duquel l'augmentation du débit d'entrée engendre une diminution de la vitesse et du débit de l'écoulement.

Ainsi, et c'est tout a fait paradoxal, alors que plus de voitures/malades entrent, le débit de sortie baisse. Deux facteurs se potentialisent et conduisent au phénomène d'encombrement.

La question que l'on se pose ici est celle de nos moyens et de la nature de notre réponse dans notre pratique quotidienne au problème de la saturation de l'encombrement de notre salle d'attente.

Encore une fois, l'affluence crée le blocage et la saturation. Le blocage amplifie lui-même à son tour la saturation. La saturation, la salle d'attente pleine renforce les blocages, ect. Quels moyens possède le clinicien seul en débutant sa garde pour lutter contre ce cercle vicieux ? En imaginant un urgentiste paré au combat, formé par les meilleurs et au mieux de son état, il peut au mieux lutter contre les effets pervers de l'encombrement et prendre en charge de manière fiable et avisée ses malades avec son rythme de croisière, le débit qui a été freiné par les évènements que nous venons de mentionner. Ainsi, au mieux, il empêche le grossissement $\mathrm{du}$ bouchon, travaillant dans des conditions hostiles. $\mathrm{Au}$ mieux...

Souvent en revanche, il est ralenti par les frictions et turbulences inhérentes à ce débit élevé, devient vite fatigué, probablement énervé. Dans ces conditions, personne ne peut dire que le médecin urgentiste est en pleine possession de ses moyens cognitifs et surtout humains pour bien appréhender les malades qui passent entre ses mains.

Le seul moyen de rompre ce cercle vicieux, la seule intervention possible en dehors d'essayer de faire son travail « au mieux malgré... » est celle de faire baisser le débit, la pression en amont. L'organisation des services d'urgences en France, assez uniforme, fait généralement trier à l'arrivée les patients par ordre de priorité. Ils doivent être vus dans l'ordre qu'impose leur situation ce qui est logique, et sécurisant. Mais cette organisation omet une donnée qui peut avoir son intérêt : le temps même de prise en charge du malade. Il se trouve que généralement, moins le motif de recours aux urgences est justifié, moins la pathologie semble urgente, et plus le temps de prise en charge médical est court.

Evidemment, un patient qui s'est coincé l'auriculaire dans une porte et s'inquiète d'un hématome sous-unguéal est classé comme étant le moins urgent. Il sera probablement vu le dernier. Cette organisation est pleinement justifiée par les impératifs de gravité des autres malades. Tant pis si Monsieur " Hématome de l'ongle " attends quatre heures pour un conseil médical : l'important est que Madame « Hémorragie digestive » soit vue au plus vite. Mais avec un peu de bon sens, on préfère réorienter Monsieur « Hématome de l'ongle » et voire Madame "Hémorragie digestive " 180 secondes plus tard.
Environ $80 \%$ des malades consultant aux urgences ne sont pas hospitalisés au décours et $16 \%$ sortent sans examens paraclinique [10]. Beaucoup d'entre eux ne nécessitent qu'une consultation rapide, un conseil, une réorientation, une réassurance. On note d'ailleurs que cette consultation peut d'autant plus être bien vécue par le patient, même rapide, si elle a lieu après une attente minimale. Après quatre heures d'attente, Monsieur " Hématome de l'ongle » peut être furieux qu'on l'ait fait « attendre quatre heures pour ça ?! ». Cela peut paraître anodin, après tout, quel intérêt porter à l'énervement de ce patient, son attente, ou celle de son voisin qui n'a pas trouvé de médecin pour lui renouveler son ordonnance... Mais diminuer d'autant le débit en amont peut permettre comme on a pu le suggérer de diminuer les frictions (« J'ai attendu quatre heures pour ça ?»), les turbulences ( Non monsieur je ne peux pas vous dire dans combien de temps le médecin pourra vous voir ») et le chaos engendrés par cette salle d'attente pleine.

Ainsi, repérer d'emblée certains patients qui vont nécessiter les soins les moins longs pourrait se révéler salutaire. La variation du nombre de passage par jour dans les SAU est faible. Ces derniers évoluent à flux tendu pour la plupart, ainsi une hausse d'activité de $10 \%$ s'ensuit facilement de la saturation ce ceux-ci. Diminuer d'autant la pression en amont grâce à la prise en charge rapide de ces $16 \%$ de patients ne nécessitant aucun acte autre qu'une consultation peut avoir pour corollaire de finalement baisser le temps d'attente des autres malades.

Il s'agit d'optimiser les conditions de travail de tous, et de rendre le fonctionnement du service plus fluide. Décaler régulièrement de quelques dizaines de minutes la prise en charge des malades sérieux (on ne parle évidemment pas ici de malades nécessitant une prise en charge immédiate) pourrait permettre une amélioration globale de leur prise en charge.

En aucun cas il ne s'agit ici de remettre en cause le fonctionnement actuel de nos SAU, la place de l'infirmière d'accueil et d'orientation ou du médecin coordonateur. En aucun cas ce changement de pratique ne se veut une diminution de la qualité des soins au profit d'une efficience de gestion de flux. En inversant simplement certains ordres de passage, tout le monde pourrait en profiter. Patient grave, patient léger, et personnel des urgences. Nicolas Belorgey publiait récemment une étude sur le temps de passage aux urgences, et notait une corrélation inverse entre le temps de passage aux urgences et le taux de reconsultation [11]. Ce résultat amène à deux commentaires majeurs pour le nuancer :

- d'une part, comme le souligne l'auteur, même si la corrélation est significative, $80 \%$ des variations du taux de retour ne sont pas expliquées par la réduction du temps de prise en charge ;

- d'autre part, il s'agit d'une étude évaluant les temps moyen de passage par médecin : les résultats s'appliquent 
donc d'avantage aux pratiques personnelles des médecins étudiés. On imagine bien que certains médecins plus consciencieux, plus exhaustifs dans leurs examens, plus systématique, ont un taux de retour inférieur.

En mécanique des fluides, l'écoulement dans une tuyère est laminaire jusqu'à une certaine limite de débit, après quoi il devient turbulent. L'écoulement d'un gaz ou d'un liquide s'accompagne d'un changement de phase lorsqu'il est soumis à trop de contraintes de débit et de résistance : le gaz se liquéfiera, le liquide se solidifiera.

Sur l'autoroute, en période de pointe, on propose aux automobilistes de réduire leur vitesse pour arriver plus tôt à destination. L'idée sous-jacente est la même : retarder un peu la prise en charge de certains malades plus lourd au profit d'une accélération de la prise en charge de malades « plus légers » pourrait être salutaire. C'est en tout cas une hypothèse qui mérite d'être testée.

\section{Références}

1. Sklar DP, Handel DA, Hoekstra J, et al (2010) The future of emergency medicine: an evolutionary perspective. Acad Med. Mar 85:490-5

2. Forero R, McCarthy S, Hillman K (2011) Access block and emergency department overcrowding. Crit Care 15:216
3. Caplan GA, Williams AJ, Daly B, et al (2004) A randomized, controlled trial of comprehensive geriatric assessment and multidisciplinary intervention after discharge of elderly from the emergency department--the DEED II study. J Am Geriatr Soc 52: $1417-23$

4. Donald KJ, Smith AN, Doherty S, et al (2005) Effect of an onsite emergency physician in a rural emergency department at night. Rural Remote Health 5:380

5. Dunn R (2003) Reduced access block causes shorter emergency department waiting times: An historical control observational study. Emerg Med (Fremantle) 15:232-8

6. Han JH, Zhou C, France DJ, et al (2007) The effect of emergency department expansion on emergency department overcrowding. Acad Emerg Med 14: 338-43

7. Levin SR, Dittus R, Aronsky D, et al (2008) Optimizing cardiology capacity to reduce emergency department boarding: a systems engineering approach. Am Heart J 156:1202-9

8. Mace SE, Graff L, Mikhail M, et al (2003) A national survey of observation units in the United States. Am J Emerg Med. 21:529-33

9. Guttmann A, Schull MJ, Vermeulen MJ, et al (2011) Association between waiting times and short term mortality and hospital admission after departure from emergency department: population based cohort study from Ontario, Canada. BMJ.342:d2983

10. Carrasco V, Beaubeau D, Direction de la recherche, des études, de l'évaluation, et des statistiques (DREES) (2003) Les usagers des urgences Premiers résultats d'une enquête nationale. Etudes et Résultats $\mathrm{N}^{\circ} 212$ (http://www.drees.sante.gouv.fr/les-usagersdes-urgences-premiers-resultats-d-une-enquete-nationale, 4728. html, dernier accès 12 Février 2013).

11. Belorgey N (2011) Réduire le temps de passage aux urgences. Actes de recherche en sciences sociales. 189:16-33 